\title{
Secretory signal peptide modification for optimized antibody-fragment expression-secretion in Leishmania tarentolae
}

Stephan $\mathrm{Klatt}^{1,2}$ and Zoltán Konthur ${ }^{1 *}$

\begin{abstract}
Background: Secretory signal peptides (SPs) are well-known sequence motifs targeting proteins for translocation across the endoplasmic reticulum membrane. After passing through the secretory pathway, most proteins are secreted to the environment. Here, we describe the modification of an expression vector containing the SP from secreted acid phosphatase 1 (SAP1) of Leishmania mexicana for optimized protein expression-secretion in the eukaryotic parasite Leishmania tarentolae with regard to recombinant antibody fragments. For experimental design the online tool SignalP was used, which predicts the presence and location of SPs and their cleavage sites in polypeptides. To evaluate the signal peptide cleavage site as well as changes of expression, SPs were N-terminally linked to single-chain Fragment variables (scFv's). The ability of $L$. tarentolae to express complex eukaryotic proteins with highly diverse post-translational modifications and its easy bacteria-like handling, makes the parasite a promising expression system for secretory proteins.

Results: We generated four vectors with different SP-sequence modifications based on in-silico analyses with SignalP in respect to cleavage probability and location, named pLTEX-2 to PLTEX-5. To evaluate their functionality, we cloned four individual scFv-fragments into the vectors and transfected all 16 constructs into L. tarentolae. Independently from the expressed SCFv, pLTEX-5 derived constructs showed the highest expression rate, followed by pLTEX-4 and pLTEX-2, whereas only low amounts of protein could be obtained from pLTEX-3 clones, indicating dysfunction of the SP. Next, we analysed the SP cleavage sites by Edman degradation. For pLTEX-2, -4 , and -5 derived scFv's, the results corresponded to in-silico predictions, whereas PLTEX-3 derived scFv's contained one additional amino-acid (AA).

Conclusions: The obtained results demonstrate the importance of SP-sequence optimization for efficient expression-secretion of scFv's. We could successfully demonstrate that minor modifications in the AA-sequence in the c-region of the natural SP from SAP1, based on in-silico predictions following the $(-3,-1)$ rule, resulted in different expression-secretion rates of the protein of interest. The yield of scFv production could be improved close to one order of magnitude. Therefore, SP-sequence optimization is a viable option to increase the overall yield of recombinant protein production.
\end{abstract}

Keywords: Secretory signal peptide (SP), SignalP, single-chain Fragment variable (scFv), Leishmania tarentolae

\footnotetext{
* Correspondence: Konthur@molgen.mpg.de

${ }^{1}$ Max Planck Institute for Molecular Genetics, Ihnestraße 63-73, 14195 Berlin, Germany

Full list of author information is available at the end of the article
}

\section{Biomed Central}

(c) 2012 Klatt and Konthur; licensee BioMed Central Ltd. This is an Open Access article distributed under the terms of the Creative Commons Attribution License (http://creativecommons.org/licenses/by/2.0), which permits unrestricted use, distribution, and reproduction in any medium, provided the original work is properly cited. 


\section{Background}

Leishmania tarentolae is a eukaryotic flagellated unicellular parasite with a broad range of applications [1-3]. An increasing field of interest is its use as a host for recombinant protein expression [4-8]. It allows complex eukaryotic protein expression at high levels compared to other eukaryotes, and has the ability to posttranslationally modify proteins. Furthermore, its easy bacteria-like handling makes the parasite a promising expression system for eukaryotic proteins. All these characteristics suggest Leishmania to be a good choice for the expression-secretion of recombinant antibody fragments.

A single-chain Fragment variable (scFv) is the smallest functional entity of a monoclonal antibody consisting of a single-polypeptide. It is composed of the variable regions of the heavy $\left(\mathrm{V}_{\mathrm{H}}\right)$ and the light $\left(\mathrm{V}_{\mathrm{L}}\right)$ chain of immunoglobulins, which are connected with a flexible amino-acid (AA) linker of varying length [9].

Antibodies, like many other proteins, are naturally secreted. For targeted protein transport, special sequence motifs are usually necessary [10-12]. Secretory signal peptides (SP) function as sorting signals. In general, they are located at the $\mathrm{N}$-terminus of proteins and their length ranges between 15-30 AAs [13]. During translocation across the endoplasmic reticulum membrane, the SP is usually cleaved off and the protein is entering the secretory pathway $[11,13,14]$. Changes of $2-4$ AAs of the SP-sequence can result in new cleavage sites and in changed expression-secretion efficiency in e.g. lactic acid bacteria [15]. Secretory leader sequence optimization has been widely applied in other organisms as well, such as E. coli [16], different yeast strains $[17,18]$, or in insect cells using the baculoviral expression system [19].

In this study, we explored the use of $L$. tarentolae as host for protein expression-secretion of four human recombinant scFv's derived from a semi-synthetic single-framework phage display antibody library [20,21]. To accommodate scFv's with efficient cleavage sites, we followed a two step strategy. First, we set out to in-silico model SP-sequences in combination with suitable restriction sites for cloning using SignalP [22]. Second, we designed appropriate vector constructs to evaluate resulting SP-sequences invivo for optimized $\mathrm{scFv}$ expression-secretion in $L$. tarentolae. As a starting point we utilised the commercial L. tarentolae protein expression-secretion vector pLEXSY-sat2 (Jena Bioscience), containing the SPsequence of secreted acid phosphatase 1 (SAP1 [UniProt:Q25332]) of L. mexicana [23]. This SP-sequence has been successfully applied in a similar or the same vector for the expression-secretion of e.g human erythropoietin [4], C-reactive protein [24] and heterotrimeric laminins [25].

\section{Results and discussion}

In-silico analysis of natural secretory signal peptides

A number of online tools are available for predicting signal peptides and corresponding cleavage sites in protein constructs based on their AA sequence [13]. In two comparative studies, the online program SignalP was identified to be the method of choice [26,27]. Thus, we applied this online tool for cleavage site prediction using an algorithm based on Hidden Markov Models (HMM) on the SP-sequence in vector pLEXSY-sat2 in combination with human $\mathrm{scFv}$ sequences.

First, we analysed the cleavage site for natural human IgG expression-secretion with its natural IgG $\mathrm{V}_{\mathrm{H}}$ leader peptide [UniProt:Q9Y298] [28] in plasma cells using SignalP 3.0 [29] (MDWTWRILFLVAAATGTHA_scFv), which resulted in a $100 \%$ cleavage prediction at the first AA of the $\mathrm{scFv}$ (Figure 1A). In parallel, we compared the cleavage site of scFv constructs with the Erwinia carotovora pectate lyase 2 (pelB) leader peptide [UniProt: P0C1C1] [30] (MKYLLPTAAAGLLLLAAQPAMA_scFv; Figure 1B). The SP of pelB is frequently used for $\mathrm{scFv}$ expression-secretion in Escherichia coli and results in the same cleavage site as during natural human $\operatorname{IgG}$ expression-secretion. Next, we analysed whether a simple transfer of a scFv into L. tarentolae expression-secretion vector pLEXSY-sat2 would yield the same results. Endogenously, the SP of SAP1 (MASRLVRVLAAAML VAAAVSVDA_SAP1) is cleaved off completely. In-silico, SignalP 3.0 assigns a probability of $\sim 95 \%$ for this cleavage event (Figure 1C). If scFv's were theoretically to be cloned directly in fusion with the SAP1 SP-sequence neglecting the necessity for a cloning site, the predicted cleavage probability is reduced to $\sim 83 \%$ (Figure 1D). In pLEXSYsat2, the $\mathrm{scFv}$ could be cloned downstream of the SPsequence into the multiple cloning site II (MCS II, underlined in the following) by using the available restriction site XbaI. This would result in the AA-sequence MASRLVRVLAAAMLVAAAVSVDAGASLD_scFv. The in-silico cleavage prediction of this construct worsens. No unique cleavage site was assigned, rather multiple sites were predicted with similar probabilities ranging only around $10-30 \%$ (Figure 1E). Further, none of the cleavage sites were predicted to yield the same cleavage product as E. coli or human cells, i.e. targeting the first AA of the scFv (glutamic acid (E); EVQLLES...), which is defined as position 1 . This is attributed to the fact that the AA composition upstream position $1(-1$ to -3$)$ has an influence on the cleavage site [31]. Additionally, at least one amino-acid is not cleaved off extending the scFv-sequence $\mathrm{N}$-terminally, which could potentially influence protein functionality. For all these reasons we conclude that the use of the pLEXSY-sat2 vector in its current state is not sensible for expression-secretion of scFv's in $L$. tarentolae. Hence, the vector needs specific 


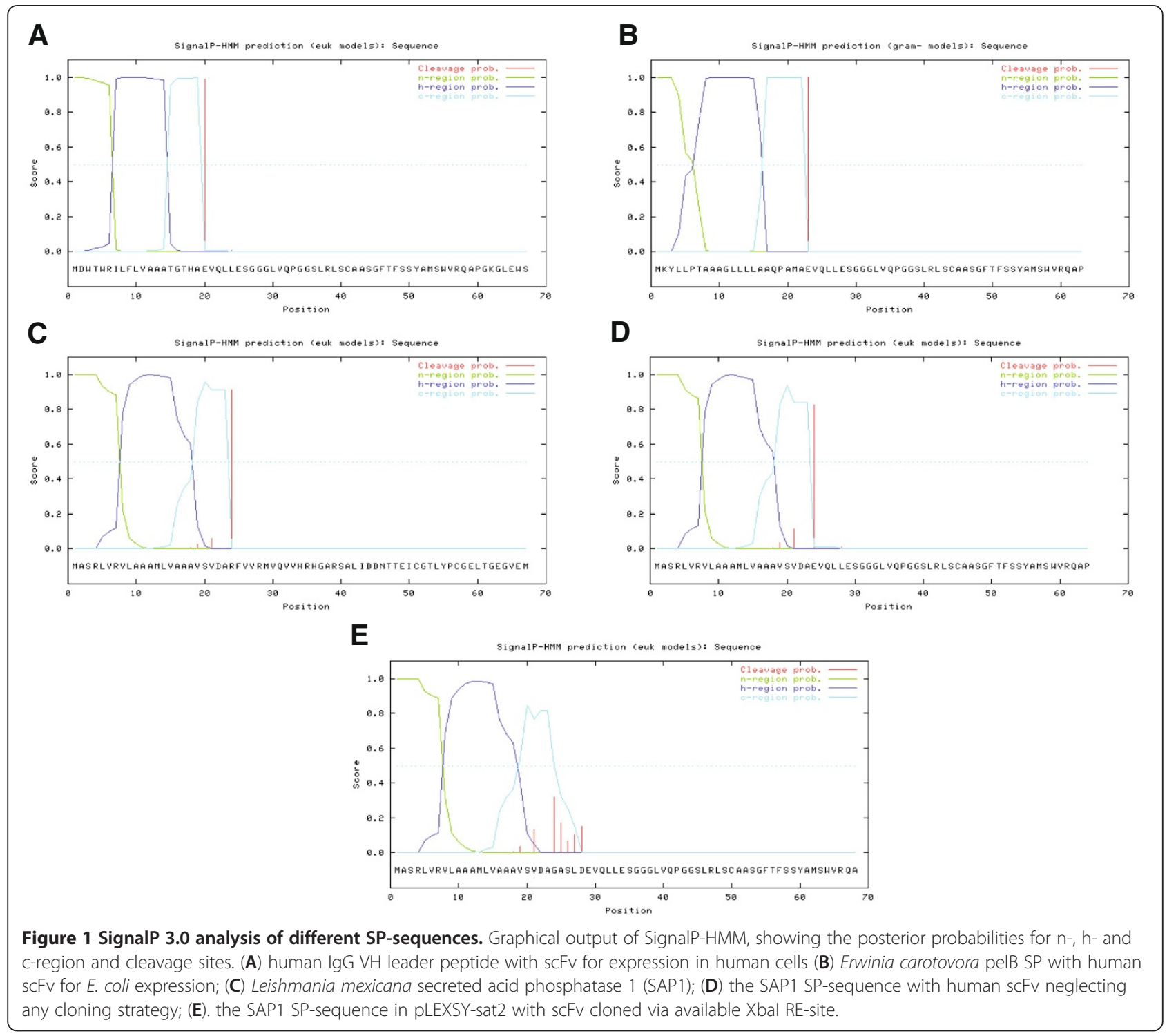

modification in regard to cleavage site as well as cleavage probability.

\section{In-silico modification of secretory signal peptide SAP1 for scFv expression}

Modification of the SP-sequence to enhance cleavage site probability at the first AA of scFv's was carried out insilico following two rationales: First, to optimize AA composition defined by the multi cloning site (MCS II) accommodating unique restriction enzyme (RE) sites for $\mathrm{scFv}$ cloning. Second, to find a site with the highest possible cleavage probability and lowest number of alternatives at the first AA residue of the scFv. Ideally, fewer cleavage sites predicted from SignalP and a higher probability of only one successful cleavage should lead to better expression-secretion results of the protein.
The scFv's in our experiments were derived from the phage display vector pIT2, which contains a unique NcoI RE-site upstream of position 1 . To enable simple cloning, the MCS II should contain a NcoI RE-site or, alternatively, a RE-site with compatible overhang, such as Pcil. Hence, in an iterative process we first set out to modify only the MCSII by introducing changes in the vector pLEXSY-sat2 on the nucleic acid level to accommodate compatible RE-sites for cloning and analysed the results in-silico applying SignalP. This approach resulted in the design of pLTEX-2, described in detail below. In a second step, we included the modification of the last AA positions of the SAP1 SP-sequence and conducted the same analyses to obtain the desired characteristics of SPsequence cleavage at position 1 . This approach led to the design of vectors pLTEX-3 to pLTEX-5 (Figure 2). 
The detailed rational for all four vectors is described in detail below.

pLTEX-2 was designed on the merit to maintain the original SP-sequence of SAP1, only exchanging the AA encoded by MCS II to accommodate a PciI restriction site. The SP-sequence will be cleaved at the identical position as in SAP1 and, hence, the $\mathrm{scFv}$ is extended $\mathrm{N}$-terminally by four AAs. pLTEX-2 has a predicted major cleavage site with a probability of $\sim 81 \%$ at the glycine $(\mathrm{G})$ residue in position -4 relative to the scFv's start and two minor cleavage sites at positions -7 and -3 with probabilities of $\sim 10 \%$ or below (Figure $2 \mathrm{~A}$ ).

The constructs pLTEX-3 to PLTEX-5 were designed to contain a SP-sequence with a high cleavage probability directly at position 1 . To achieve this, the natural SAP1 $\mathrm{SP}$-sequence was shortened by the two AA residues aspartic acid (D) and alanine (A), corresponding to positions -6 and -5 in pLTEX-2. At the same time we introduced variations in the AA residue at position -3 by incorporating asparagine $(\mathrm{N})$, threonine $(\mathrm{T})$ and alanine (A), respectively.
pLTEX-3 contains an asparagine residue at position -3, a predicted major cleavage site with a probability of $\sim 61 \%$ at position 1 as well as two additional cleavage sites upstream with lower probabilities $(\sim 21 \%$ and $\sim 10 \%$; Figure 2B).

pLTEX-4 and pLTEX-5 contain a threonine or alanine residue at position -3 and their predicted major cleavage sites with a probability of close to $95 \%$ is at position 1 directly. Both constructs have additional predicted minor cleavage sites with probabilities of below $\sim 5 \%$ (Figure 2C,D). The additional modification in pLTEX-4 and pLTEX-5 at the second N-terminal position of the SP-sequence (A to T) was introduced to abolish a NcoI REsite in the vector and is not considered essential for the functionality of the SP [32].

\section{Generation of expression-secretion vectors pLTEX-1 to pLTEX-5}

Novel vectors were designed based on the above considerations for AA composition of SP-sequences. First, the general transfection strategy in $L$. tarentolae was assessed.

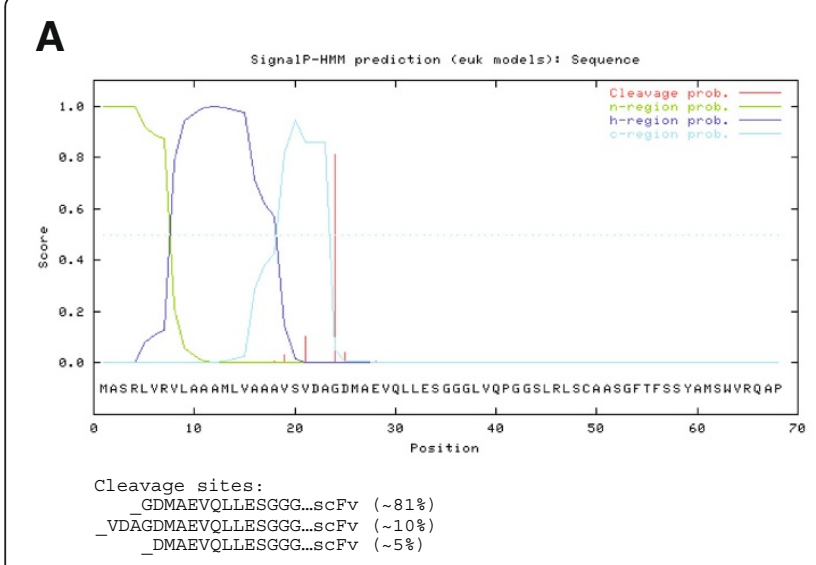

B

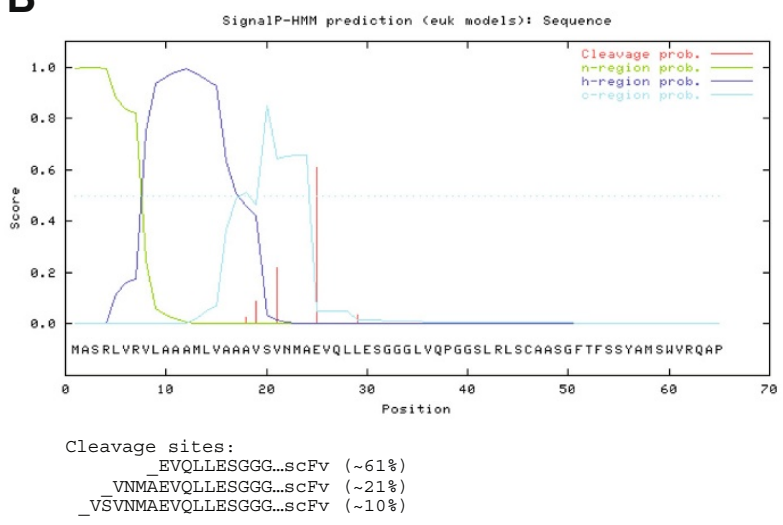

C

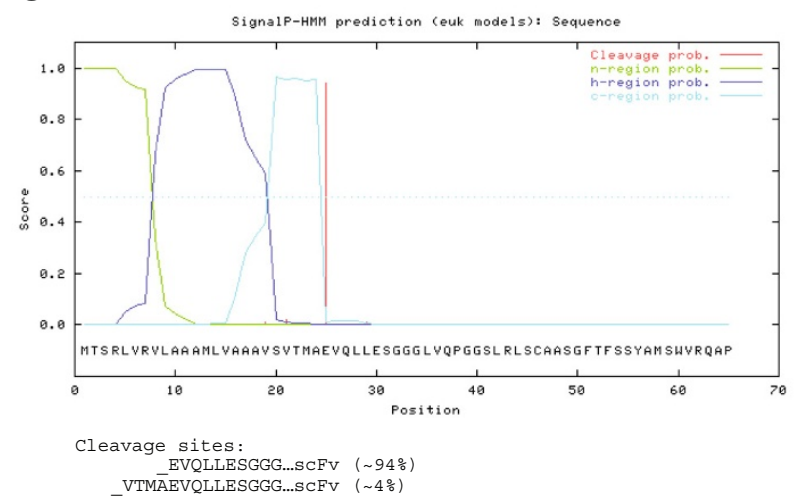

D

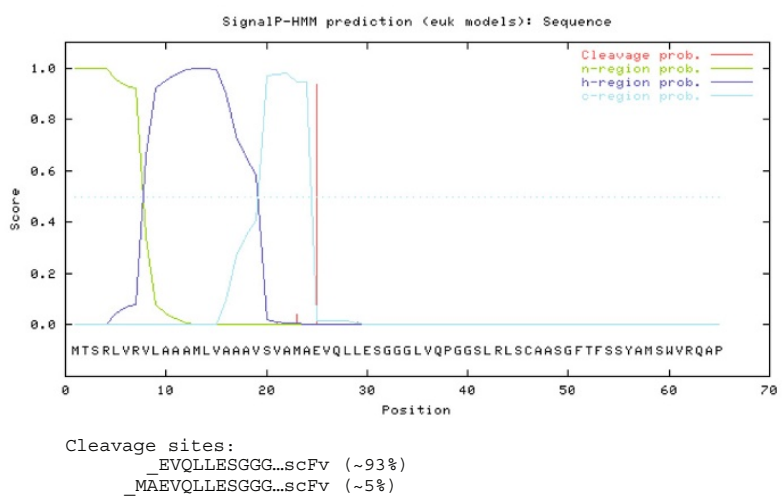

Figure 2 SignalP 3.0 analysis of novel pLTEX vectors with a scFv. Graphical output of SignalP-HMM, showing the posterior probabilities for $\mathrm{n}$-, $\mathrm{h}$ - and c-region and cleavage sites of pLTEX vectors with scFv for (A) pLTEX-2 (B) pLTEX-3, (C) pLTEX-4 and (D) pLTEX-5. Alternative cleavage sites and respective probabilities are displayed. 
To obtain stable expression of recombinant protein a linearised construct needs to be transfected to allow chromosomal integration into a predefined locus by homologous recombination [4]. The original pLEXSY-sat2 vector (Jena Bioscience) is linearised by RE SwaI (Figure 3A). However, all scFv's used in this study contain a conserved SwaI REsite. Therefore we replaced SwaI with EcoRV. At the same time we reduced the pBluescript II KS (-) backbone of the vector by $1.1 \mathrm{~kb}$ to enhance transformation efficiency in $E$. coli generating vector pLTEX-1 (Figure 3A). Note, that pLTEX-1 in its current state is not suitable for scFv expression according to our in-silico analysis alike pLEXSYsat2, since multiple cleavage sites with poor cleavage probabilities are anticipated. Next, the cloning strategy for $\mathrm{scFv}$ was determined. MCS I and MCS II were analysed for the availability of compatible restriction sites and modified to allow cloning of scFv's via NcoI and NotI. During the same process, modified SP-sequences were introduced using specific primer-sets creating expressionsecretion vectors pLTEX-2 to pLTEX-5 (Figure 3B).

In pLTEX-2 and pLTEX-3, a PciI RE-site was introduced into MCS II, since PciI produces a compatible overhang to NcoI. In vectors pLTEX-4 and pLTEX-5, a NcoI RE-site was introduced in MCS II and at the same time another abolished in MCS I.

\section{Analysis of vector-dependent scFv expression rates in vivo}

To test the expression-secretion efficiency of the new vectors pLTEX-2 to pLTEX-5, four independent scFv's were cloned into each vector and transfected into $L$. tarentolae. Stable integration of the expression cassette into

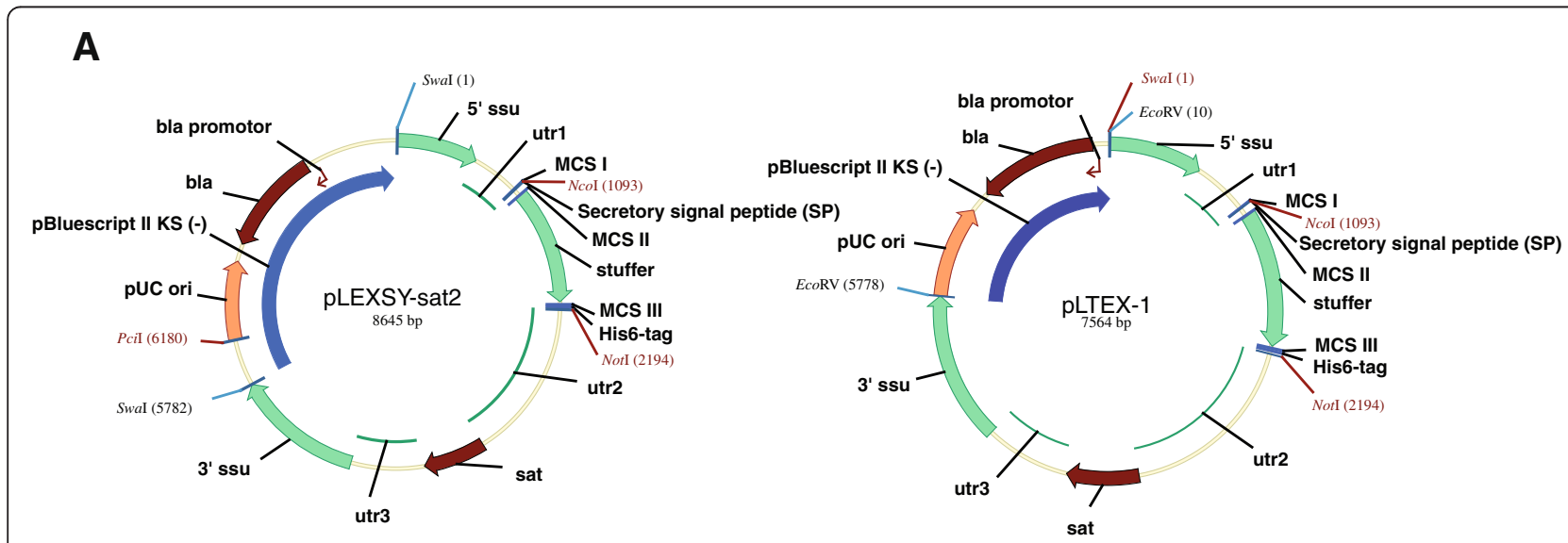

B

magnown

pLEXSY-sat2 CACCAGATCT GCCATGGCCT CGAGGCTCGT CCGTGTGCTG GCCGCCGCCA TGCTGGTTGC AGCGGCCGTG TCGGTCGACG CTGGCGCCTC TCTAGACACA

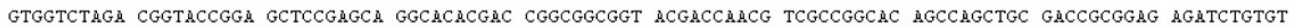

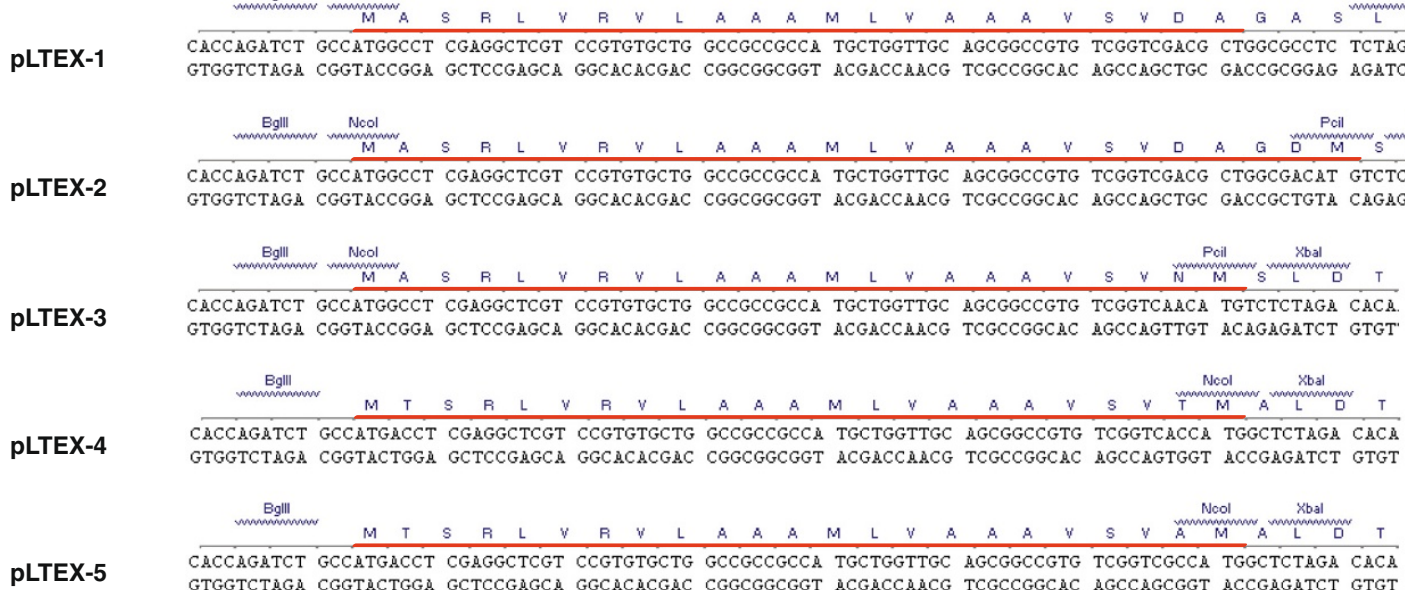

Figure 3 Description of vectors pLEXSY-sat2 and pLTEX-1 to pLTEX-5. (A) Full vector maps of pLEXSY-sat2 and pLTEX-1. pLEXSY-sat2 (Jena Bioscience) was used to generate pLTEX-1 with a reduced pBluescript II KS (-) backbone and different RE-sites for linearization (Swal to EcoRV). (B) Differences in the SP-sequences and MCS II of vectors pLEXSY-sat2 and pLTEX-1 to pLTEX-5. Protein sequence of SP's are underlined with red. 
the host chromosome was analysed by genomic PCR and could be verified for all 16 constructs (data not shown).

In all pLTEX vectors, protein expression occurs constitutively and was tested for up to four clones per construct. For each construct, the clones showed similar expression levels according to SDS-PAGE analysis and no difference in expression-secretion between singleclones and multi-clones (collection of unknown number of single-clones) from the same vector was observed (data not shown).

First, we analysed the culture supernatant of all 16 different $\mathrm{scFv}$ constructs by Western blot for the presence of $\mathrm{scFv}$ and observed concentration differences (Figure 4A). Independent of which $\mathrm{scFv}$ was cloned, most protein could be detected in supernatants of clones expressed in pLTEX-5 followed by pLTEX-4, pLTEX-2 and pLTEX-3. The nature of SP-sequence and its corresponding cleavage site can influence the expression-secretion efficiency [15] and has a direct influence on the expression level of scFv's.

Next, we purified scFv from the culture supernatant of all 16 clones by immobilised metal-ion chromatography (IMAC) and analysed the data by Coomassiedyed SDS-PAGE (Figure 4B). As expected, the same order of expression efficiency was observed and the intensity of the protein band corresponding to the scFv $(\sim 27 \quad \mathrm{kDa})$ was quantified densitometrically (Figure 4C). The highest expression rate for scFv's was recorded from clones in vector pLTEX-5, with a median expression of $3.83 \mathrm{mg} / \mathrm{L}$. Clones from vectors pLTEX-4 and pLTEX-2 had a median expression rate of $1.05 \mathrm{mg} / \mathrm{L}$ and $0.53 \mathrm{mg} / \mathrm{L}$, respectively. The lowest median expression rate was observed in clones from vector pLTEX-3 $(0.04 \mathrm{mg} / \mathrm{L})$.
The observed expression-secretion efficiencies are in good agreement with our knowledge on SP-sequences, formulated as the signal peptidase recognition site or $(-3,-1)$ rule [33] based on the analysis of 78 different natural eukaryotic signal sequences [31]. The preference of a small neutral AA in position -1 and exclusion of a certain large polar, aromatic and charged AA in position -3 can be explained by the nature of the signal peptidase I, which is mainly responsible for SP cleavage. The following ranking was deduced according a 'best fit' analysis for AA and signal peptidase I combination for position -1 : A $>$ G,S $>C, T>Q$; for position -3 : $A>V>$ $\mathrm{C}, \mathrm{S}, \mathrm{T}>\mathrm{L}, \mathrm{I}>\mathrm{G}$ [33].

In concordance with this, all our vectors follow this general rule. In detail, our choice for alanine in position -1 respectively to the cleavage site in all pLTEX vectors is optimal. However, the AAs in position -3 are different. Here, pLTEX-5 and pLTEX-4 have the preferred small neutral AA (A, T) whereas pLTEX-2 has a hydrophobic AA (V). The SP-sequence of pLTEX-3 has a large polar AA (N) in position -3 resulting in poor expression, possibly due to steric hindrance within the active site of signal peptidase I resulting in low cleavage efficiency. The expression level is in good agreement with the order of the AA observed in position -3 with alanine giving the best expressionsecretion rate.

Finally, we analysed the AA-composition of the Nterminus of all 16 obtained scFv's by Edman degradation [34]. In pLTEX-2 the SP is cleaved off at the predicted glycine residue four AA downstream of the first AA (E) of the scFv. For vectors pLTEX-4 and pLTEX-5 the $\mathrm{N}$-terminus also follows the prediction. The SP is cleaved off at the predicted site, which is glutamic acid (E). Additionally, a second cleavage site could be detected at position -2 , extending the scFv's by two AAs; methionine
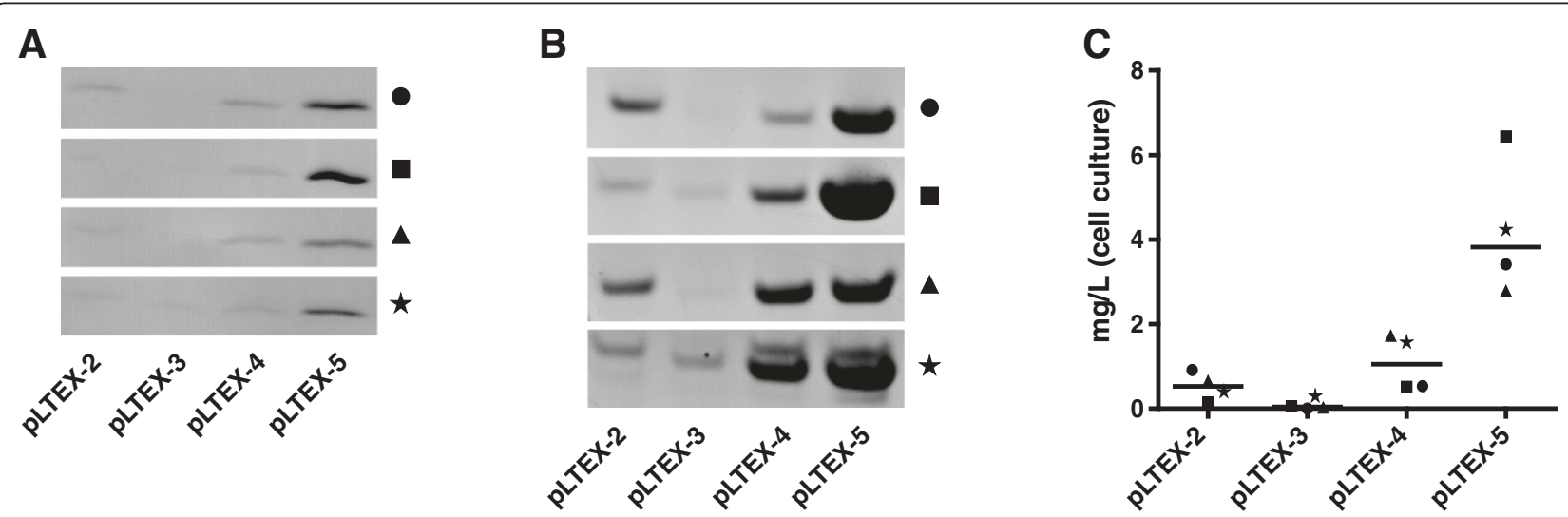

Figure 4 Expression-secretion analysis of 16 scFv's in vectors pLTEX-2 to pLTEX-5. (A) Western blot analysis of scFv's in culture supernatant. Detection of scFv's with Protein L-HRP (1:5000). (B) SDS-PAGE analysis of Ni-NTA purified scFv's from $100 \mathrm{ml}$ cultures. Proteins are separated on 4-12\% Bis-Tris gels (Invitrogen). (C) Protein yield from $100 \mathrm{ml}$ culture volumes grown for $72 \mathrm{~h}$ of IMAC-purified scFv's determined in duplicate by densitometrical analysis on SDS-PAGE. Black circles, squares, triangles and stars represent scFv-1 to -4 , respectively. 
and alanine (MA). Notably, the AA-composition in position -5 and position -3 also fulfils the signal peptidase recognition site rule, but with low efficiency. The observed frequency of this cleavage isotype has become visible after digesting large quantities of the protein (data not shown).

Surprisingly, the expected in-silico cleavage site at the first AA of the scFv could not be confirmed in the samples derived from vector pLTEX-3. The SP was cleaved one AA downstream at an alanine residue extending the scFv's. We propose that the offset of signal peptidase I occurs as a consequence of the unfavourable AA composition downstream of position 1 , resulting in inefficient cleavage explaining the low expression-secretion yield.

\section{Conclusions}

To our knowledge, this study represents the first in detail analysis of a signal peptidase recognition site in $L$. tarentolae. We could successfully demonstrate that minor modifications in the AA-sequence of the natural SP from SAP1 based on in-silico predictions, following the $(-3,-1)$ rule resulted in different expression-secretion rates of the protein of interest. Using these general principals the yield of $\mathrm{scFv}$ production could be improved close to one order of magnitude.

\section{Methods}

Generation of expression vectors pLTEX-1 to pLTEX-5

All plasmids and primers used in this study are listed in Tables 1 and 2, respectively. Vector pLTEX-1 (Figure 3A) was generated in three steps: First, the pBluescript II KS (-) backbone (ori for E. coli) from pLEXSY-sat2 (Jena Bioscience) was reduced from $2.86 \mathrm{~kb}$ to $1.82 \mathrm{~kb}$ by PCR amplification using primer 1 and 2 . The primers added EcoRV restriction sites to both ends of amplicon 1 . Secondly, the chromosomal integration cassette was PCR amplified using primers 3 and 4 (5783 bp) from pLEXSYsat2. Again, the primers added EcoRV restriction site to both ends creating amplicon 2. Next, amplicon 1 and 2 were digested with EcoRV, ligated, transformed into electrocompetent DH10B cells (Invitrogen) and plated on Ampicillin-containing agar-plates for clonal selection. The obtained vector was named pLTEX-1P (P for precursor). In a final step, the expression cassette was exchanged in pLTEX-1P with a parental fragment from vector pLEXSY-sat2. For this, both vectors were digested with

Table 1 Vectors and constructs used in this study

\begin{tabular}{|c|c|c|}
\hline Name & Characteristics & Reference \\
\hline pLEXSY-sat2 & $\begin{array}{l}\text { Expression vector pLEXSY-sat2 with sat gene (streptothricine acetyltransferase) } \\
\text { allowing selection of recombinant LEXSY strains with antibiotic NTC; } \\
\text { and Amp for DH10B }\end{array}$ & $\begin{array}{l}\text { Jena Bioscience } \\
\text { (Cat.-No. EGE-234) }\end{array}$ \\
\hline pLTEX-1 & $\begin{array}{l}\text { Based on pLEXSY-sat2; with reduced pBlueScript backbone and changed RE-site } \\
\text { for vector linearization prior transfection (Swal to EcoRV) }\end{array}$ & this study \\
\hline pLTEX-2 & based on pLTEX-1; changed SP sequence & this study \\
\hline pLTEX-3 & based on pLTEX-1; changed SP sequence & this study \\
\hline pLTEX-4 & based on pLTEX-1; changed SP sequence & this study \\
\hline pLTEX-5 & based on pLTEX-1; changed SP sequence & this study \\
\hline pLTEX-2_scFv-1 & Vector for expression-secretion of scFv-1 & this study \\
\hline pLTEX-3_scFv-1 & Vector for expression-secretion of scFv-1 & this study \\
\hline pLTEX-4_scFv-1 & Vector for expression-secretion of scFv-1 & this study \\
\hline$\overline{p L T E X-5 \_s c F v-1}$ & Vector for expression-secretion of scFv-1 & this study \\
\hline pLTEX-2_scFv-2 & Vector for expression-secretion of scFv-2 & this study \\
\hline pLTEX-3_scFv-2 & Vector for expression-secretion of scFv-2 & this study \\
\hline pLTEX-4_scFv-2 & Vector for expression-secretion of scFv-2 & this study \\
\hline pLTEX-5_scFv-2 & Vector for expression-secretion of scFv-2 & this study \\
\hline pLTEX-2_scFv-3 & Vector for expression-secretion of scFv-3 & this study \\
\hline pLTEX-3_scFv-3 & Vector for expression-secretion of scFv-3 & this study \\
\hline pLTEX-4_scFv-3 & Vector for expression-secretion of scFv-3 & this study \\
\hline pLTEX-5_scFv-3 & Vector for expression-secretion of scFv-3 & this study \\
\hline pLTEX-2_scFv-4 & Vector for expression-secretion of scFv-4 & this study \\
\hline pLTEX-3_scFv-4 & Vector for expression-secretion of scFv-4 & this study \\
\hline pLTEX-4_scFv-4 & Vector for expression-secretion of scFv-4 & this study \\
\hline pLTEX-5_scFv-4 & Vector for expression-secretion of scFv-4 & this study \\
\hline
\end{tabular}


Table 2 Oligonucleotides used in this study

\begin{tabular}{ll}
\hline Name & Sequence (5' to $\left.\mathbf{3}^{\prime}\right)$ \\
\hline (1) pre-pBR322-ori-EcoRV & CGGATATCTGAGCAAAAGGCCAGCAAAA \\
\hline (2) pre-blaP-Swal-EcoRV & CGGATATCCAATTAAATGCGGAACCCCTATTTGTTTATT \\
\hline (3) 5_EcoRV_5_ssu & TTGGATATCTTGGCGAAACGCC \\
\hline (4) EcoRV_3_ssu & GATTAGATATCGGTGAACTTTCGGG \\
\hline (5) Pci_linker_sat4 & GCTCTAGAGACATGTCGCCAGCGTCGACCG \\
\hline (6) Pci_linker_sat5 & GCTCTAGAGACATGTTGACCGACACGGCCGCTG \\
\hline (7) BgllI_linker_sat6 LEXSY_A264 & GAAGATCTGCCATGACCTCGAGGCTCGTC \\
\hline (9) Ncol_linker_sat6 & CATCTATAGAGAAGTACACGTAAAAG \\
\hline (10) Ncol_linker_sat7 & GCTCTAGAGCCATGGTGACCGACACGGCCGCTG \\
\hline (11) LMB3 & GCTCTAGAGCCATGGCGACCGACACGGCCGCTG \\
\hline (12) LEXSY_scFv_Kpnl & CAG GAA ACA GCT ATG AC \\
\hline
\end{tabular}

BsrGI and BamHI, respective fragments were gelpurified, ligated and transformed into DH10B cells to generate the expression vector pLTEX-1. Vectors pLTEX-2 to -5 are based on pLTEX-1 and only differ in the SP-sequence. Vector pLTEX-2 was generated from pLTEX-1 and amplicon 3, which was a PCR fragment from pLEXSY-sat2 using primers 3 and 5. Both, PCR fragment and pLTEX-1 were digested with BamHI and $\mathrm{XbaI}$, ligated together and transformed into DH10B cells to generate pLTEX-2. Vectors pLTEX-1 and pLTEX-2 only differ in MCS II that now contains a RE-site for PciI. Vector pLTEX-3 was generated as described for pLTEX-2, except using primer 6 instead of 5 . For the generation of pLTEX-4, another precursor (pLTEX-4P) was necessary. First, a PCR amplicon using primers 7 (adding cutting site BglII) and 8 was generated on pLEXSY-sat2 as template. The PCR-fragment and pLTEX-1 were then digested with BgIII and KpnI, ligated together and transformed into DH10B to generate pLTEX4P. In a second step, a PCR amplicon using primers 3 and 9 (adding cutting site NcoI) was generated on pLTEX-4P. This PCR-fragment and pLTEX-1 were digested with BamHI and XbaI, ligated and transformed into DH10B cells to generate pLTEX-4. Vector pLTEX-5 was constructed starting from pLTEX-4P by generating a PCR amplicon using primers 3 and 10 (adding cutting site NcoI). Both, PCR-fragment and pLTEX-1 were digested with BamHI and XbaI, ligated and transformed into DH10B cells to generate pLTEX-5. All new vectors were sequence-verified by DNA sequencing applying the Sanger method.

\section{Cloning of antibody fragments}

Individual scFv's were PCR-amplified from the original clones in vector pIT2 using primer 11 and 12 (adding cutting site KpnI). The PCR-fragments (729 bp) were digested with $\mathrm{NcoI}$ and KpnI for cloning, ligated into cut vector backbones and transformed into DH10B. Vectors
pLTEX-2 and pLTEX-3 were digested with Pcil and KpnI, while vectors pLTEX-4 and pLTEX-5 were digested with $\mathrm{NcoI}+\mathrm{KpnI}$. Transformants were picked from $A m p^{R}$ agar-plates and sequence verified. Altogether, 16 different scFv-containing vector constructs were designed (Table 1).

\section{E. coli growth conditions and cell transformation}

E. coli strain 'ElectroMAX ${ }^{\mathrm{Tm}} \mathrm{DH} 10 \mathrm{~B}^{\mathrm{Tm}}$ (Invitrogen; Cat.-No. $18290-015)$ was grown at $37^{\circ} \mathrm{C}$ with $160-180 \mathrm{rpm}$ in liquid 2YT growth medium [16 g/l bacto-tryptone (Becton, Dickinson and Company), $10 \mathrm{~g} / \mathrm{l}$ bacto-yeast extract (Becton, Dickinson and Company), $5 \mathrm{~g} / \mathrm{l} \mathrm{NaCl}$ (Calbiochem); resuspended in Millipore water] supplemented with ampicillin (100 $\mu \mathrm{g} / \mathrm{ml}$; Roth), if necessary. Electro-competent cells were transformed with $1 \mu \mathrm{l}$ (50-200 ng/ $\mu \mathrm{l}$ ) purified plasmid-DNA (QIAprep Spin Miniprep Kit; Qiagen) using the 'Micropulser Electroporator' (BioRad) at $1.8 \mathrm{kV}, 5 \mathrm{msec}$. For clonal selection, agar-plates were prepared by adding $15 \mathrm{~g} / \mathrm{L}$ bacto-agar-agar (Becton, Dickinson and Company) to 2YT growth medium, plating cells and incubating them over night at $37^{\circ} \mathrm{C}$.

\section{L. tarentolae growth conditions and stable transfection}

L. tarentolae strain P10 (Jena Bioscience) was grown at $26^{\circ} \mathrm{C}$ under standard air-supply in the dark in LEXSY BHI liquid medium (Jena Bioscience) with porcine Hemin, penicillin/streptomycin (Pen/Strep) and Nourseothricin (NTC; $100 \mu \mathrm{g} / \mathrm{ml}$ ), if necessary (all additives: Jena Bioscience). Strain P10 was passaged twice a week by inoculation of new growth medium in flat flaks (static) with a dilution of 1:30. Electroporation of cells was carried out in a prechilled $2 \mathrm{~mm}$ electroporation $\mathrm{cu}-$ vette using a Gene Pulser II with pulse controller and capacitance extender plus (Biorad). For transfection, 5$10 \mu \mathrm{l}$ purified and linearized plasmid-DNA (50- 
$150 \mathrm{ng} / \mu \mathrm{l})$ was added to 390-395 $\mu$ l densely grown cells (over night as agitated suspension culture (125 rpm); $\mathrm{OD}_{600}>1.5$ ). Cells were pulsed once at $0.45 \mathrm{kV}$ and $450 \mu \mathrm{F}$ to get 5-6 msec pulsing time with $\sim 0.45 \mathrm{kV}$ and $\sim 20 \Omega$, chilled shortly and cultured stationary over night at $26^{\circ} \mathrm{C}$. On the next day, the majority of the culture was dispensed onto LEXSY BHI agar plates, while the rest was cultivated in flat flaks with new selection pressure (NTC) to get multi-clone cultures. After 6-10 days, single clones were picked and grown in liquid culture by continuously increasing the volume from $0.1 \mathrm{ml}$ to $10 \mathrm{ml}$ in LEXSY BHI growth medium. Finally, transfection success was verified for integration of the expression cassette into the host chromosome by analytical PCR as described [4].

\section{Production of scFv's expressed in Leishmania tarentolae}

Positive transfectants were used for scFv-production in $100 \mathrm{ml}$ of LEXSY BHI medium containing Hemin, Pen/ Strep and NTC. For this, medium was inoculated 1:30 with densely grown cultures and cultivated in agitated suspension culture $(125 \mathrm{rpm})$ for $2-3$ days at $26^{\circ} \mathrm{C}$ until $\mathrm{OD}_{600}$ 2.5-4.5 was reached. Cells were harvested by centrifugation for $18 \mathrm{~min}$, at $1811 \mathrm{~g}$ and $4^{\circ} \mathrm{C}$. Pellet and supernatant were separated. $10 \mathrm{ml} / \mathrm{l}$ PMSF (0.1molar; Phenylmethanesulfonyl fluoride, Sigma-Aldrich; inhibits proteases) and $1 \mathrm{ml} \mathrm{Ni-NTA}$ agarose (Invitrogen) were added to $100 \mathrm{ml}$ supernatant and rotated for 2.5-3 h at $4^{\circ} \mathrm{C}$. Ni-NTA was washed $2 \mathrm{x}$ with $5 \mathrm{ml}$ washing buffer (50 mM NaH${ }_{2} \mathrm{PO}_{4}, 300 \mathrm{mM} \mathrm{NaCl}, 20 \mathrm{mM}$ imidazole) and elution was carried out $4 \mathrm{x}$ with $1 \mathrm{ml}$ elution buffer (50 mM NaH $\mathrm{PO}_{4}, 300 \mathrm{mM} \mathrm{NaCl}, 250 \mathrm{mM}$ imidazole). All centrifugation steps were performed for $5 \mathrm{~min}$, at $652 \mathrm{~g}$ and $4^{\circ} \mathrm{C}$. Elution fractions were pooled and analysed on 4-12\% Bis-Tris SDS-PAGE (Invitrogen) and western blot after electrophoretical transfer to nitrocellulose membrane. Detection of scFv's was carried out using Protein L-HRP (Pierce) diluted 1:5000 in 2\% milk powder in PBS with $0.1 \%$ Tween 20 and subsequent incubation with CN/DAB substrate (Pierce). The protein concentration was determined by gel densitometric analysis of the $\mathrm{scFv}$ bands in Coomassie-dyed SDS-PAGE using ImageJ (Version $1.45 \mathrm{~s}$ ). As reference, a calibration curve on the basis of a quantifiable protein in a marker (Precision Plus Unstained Ladder, $25 \mathrm{kDa}$ band, 0.12 $1.2 \mu \mathrm{g}$ protein) or a scFv expressed in E. coli was established and used for calculating the protein concentration applying linear regression equation. N-terminal scFv's analysis by Edman degradation was undertaken by Proteome Factory (Berlin, Germany).

\section{Abbreviations}

AA: Amino-acid; HMM: Hidden Markov Model; LEXSY BHI: Leishmania expression system brain-heart fusion medium I; MCS: Multiple-cloning site; NTC: Nourseothricin; Pen/Strep: Penicillin/Streptomycin; RE: Restriction enzyme; SAP1: Secreted acid phosphatase 1; scFv: Single-chain fragment variable; SP: Secretory signal peptide.

\section{Competing interests}

Both authors declare that they have no competing interests.

\section{Authors' contributions}

SK has performed the experiments. ZK has conceived the study. SK and ZK have designed the experiments and written the manuscript. Both authors read and approved the final manuscript.

\section{Acknowledgments}

The authors acknowledge support by the German Federal Ministry for Education and Research (BMBF) through Grant No. 0315448B and the Max Planck Society for the Advancement of Sciences. We thank Dr. Thomas Schirrmann for his advice in gel-densitometry and Dr. Jonathan Woodsmith for critical reading of the manuscript.

\section{Author details}

${ }^{1}$ Max Planck Institute for Molecular Genetics, Ihnestraße 63-73, 14195 Berlin, Germany. ${ }^{2}$ Freie Universität Berlin, Faculty of Biology, Chemistry and Pharmacy, Takustraße 3, 14195 Berlin, Germany.

Received: 15 February 2012 Accepted: 1 July 2012

Published: 25 July 2012

\section{References}

1. Basak A, Shervani NJ, Mbikay M, Kolajova M: Recombinant proprotein convertase 4 (PC4) from Leishmania tarentolae expression system: purification, biochemical study and inhibitor design. Protein Expr Purif 2008, 60(2):117-126.

2. Breton M, Zhao C, Ouellette M, Tremblay MJ, Papadopoulou B: A recombinant non-pathogenic Leishmania vaccine expressing human immunodeficiency virus 1 (HIV-1) Gag elicits cell-mediated immunity in mice and decreases HIV-1 replication in human tonsillar tissue following exposure to HIV-1 infection. J Gen Virol 2007, 88(Pt 1):217-225.

3. Yakovich AJ, Ragone FL, Alfonzo JD, Sackett DL, Werbovetz KA: Leishmania tarentolae: purification and characterization of tubulin and its suitability for antileishmanial drug screening. Exp Parasitol 2006, 114(4):289-296.

4. Breitling R, Klingner S, Callewaert N, Pietrucha R, Geyer A, Ehrlich G, Hartung R, Muller A, Contreras R, Beverley SM, et al: Non-pathogenic trypanosomatid protozoa as a platform for protein research and production. Protein Expr Purif 2002, 25(2):209-218.

5. Fritsche C, Sitz M, Weiland N, Breitling R, Pohl HD: Characterization of the growth behavior of Leishmania tarentolae: a new expression system for recombinant proteins. J Basic Microbiol 2007, 47(5):384-393.

6. Fritsche $C$, Sitz M, Wolf M, Pohl HD: Development of a defined medium for heterologous expression in Leishmania tarentolae. J Basic Microbiol 2008, 48(6):488-495.

7. Kushnir S, Gase K, Breitling R, Alexandrov K: Development of an inducible protein expression system based on the protozoan host Leishmania tarentolae. Protein Expr Purif 2005, 42(1):37-46.

8. Mureev S, Kushnir S, Kolesnikov AA, Breitling R, Alexandrov K: Construction and analysis of Leishmania tarentolae transgenic strains free of selection markers. Mol Biochem Parasitol 2007, 155(2):71-83.

9. Bird RE, Hardman KD, Jacobson JW, Johnson S, Kaufman BM, Lee SM, Lee T, Pope SH, Riordan GS, Whitlow M: Single-chain antigen-binding proteins. Science 1988, 242(4877):423-426.

10. Rapoport TA: Transport of proteins across the endoplasmic reticulum membrane. Science 1992, 258(5084):931-936.

11. von Heijne G: The signal peptide. J Membr Biol 1990, 115(3):195-201.

12. von Heijne G: Life and death of a signal peptide. Nature 1998, 396(6707):111-113.

13. Emanuelsson $\mathrm{O}$, Brunak S, von Heijne G, Nielsen $\mathrm{H}$ : Locating proteins in the cell using TargetP, SignalP and related tools. Nat Protoc 2007, 2(4):953-971.

14. Sakaguchi M: Eukaryotic protein secretion. Curr Opin Biotechnol 1997 8(5):595-601.

15. Borrero J, Jimenez JJ, Gutiez L, Herranz C, Cintas LM, Hernandez PE: Protein expression vector and secretion signal peptide optimization to drive the 
production, secretion, and functional expression of the bacteriocin enterocin A in lactic acid bacteria. J Biotechnol 2011, 156(1):76-86.

16. Jonet MA, Mahadi NM, Murad AM, Rabu A, Bakar FD, Rahim RA, Low KO, Illias RM: Optimization of a heterologous signal peptide by site-directed mutagenesis for improved secretion of recombinant proteins in Escherichia coli. J Mol Microbiol Biotechnol 2012, 22(1):48-58.

17. Liu FC, Chen HL, Chong KY, Hsu AL, Chen CM: Production of recombinant porcine colipase secreted by Pichia pastoris and its application to improve dietary fat digestion and growth of postweaning piglets. Biotechnol Prog 2008, 24(6):1333-1341.

18. Rakestraw JA, Sazinsky SL, Piatesi A, Antipov E, Wittrup KD: Directed evolution of a secretory leader for the improved expression of heterologous proteins and full-length antibodies in Saccharomyces cerevisiae. Biotechnol Bioeng 2009, 103(6):1192-1201.

19. Futatsumori-Sugai M, Tsumoto K: Signal peptide design for improving recombinant protein secretion in the baculovirus expression vector system. Biochem Biophys Res Commun 2009, 391(1):931-935.

20. de Wildt RM, Mundy CR, Gorick BD, Tomlinson IM: Antibody arrays for high-throughput screening of antibody-antigen interactions. Nat Biotechnol 2000, 18(9):989-994.

21. Holt LJ, Bussow K, Walter G, Tomlinson IM: By-passing selection: direct screening for antibody-antigen interactions using protein arrays. Nucleic Acids Res 2000, 28(15):E72.

22. Nielsen $\mathrm{H}$, Engelbrecht J, Brunak S, von Heijne G: A neural network method for identification of prokaryotic and eukaryotic signal peptides and prediction of their cleavage sites. Int I Neural Syst 1997, 8(5-6):581-599.

23. Wiese M, $\| \mathrm{g}$, Lottspeich $F$, Overath P: Ser/Thr-rich repetitive motifs as targets for phosphoglycan modifications in Leishmania mexicana secreted acid phosphatase. EMBO J 1995, 14(6):1067-1074.

24. Dortay H, Schmockel SM, Fettke J, Mueller-Roeber B: Expression of human c-reactive protein in different systems and its purification from Leishmania tarentolae. Protein Expr Purif 2011, 78(1):55-60.

25. Sugino M, Niimi T: Expression of multisubunit proteins in Leishmania tarentolae. Methods Mol Biol 2012, 824:317-325.

26. Choo KH, Tan TW, Ranganathan S: A comprehensive assessment of $\mathrm{N}$-terminal signal peptides prediction methods. BMC Bioinforma 2009, 10(Suppl 15):S2.

27. Petersen TN, Brunak S, von Heijne G, Nielsen H: SignalP 4.0: discriminating signal peptides from transmembrane regions. Nat Methods 2011, 8(10):785-786.

28. Jacquemin MG, Desqueper BG, Benhida A, Vander Elst L, Hoylaerts MF, Bakkus M, Thielemans K, Arnout J, Peerlinck K, Gilles JG, et al: Mechanism and kinetics of factor VIII inactivation: study with an IgG4 monoclonal antibody derived from a hemophilia A patient with inhibitor. Blood 1998, 92(2):496-506

29. Bendtsen JD, Nielsen H, von Heijne G, Brunak S: Improved prediction of signal peptides: SignalP 3.0. J Mol Biol 2004, 340(4):783-795.

30. Lei SP, Lin HC, Wang SS, Callaway J, Wilcox G: Characterization of the Erwinia carotovora pelB gene and its product pectate lyase. J Bacteriol 1987, 169(9):4379-4383.

31. von Heijne G: Patterns of amino acids near signal-sequence cleavage sites. Eur J Biochem 1983, 133(1):17-21.

32. von Heijne $\mathrm{G}$ : Analysis of the distribution of charged residues in the $\mathrm{N}$-terminal region of signal sequences: implications for protein export in prokaryotic and eukaryotic cells. EMBO J 1984, 3(10):2315-2318.

33. von Heijne G: How signal sequences maintain cleavage specificity. J Mol Biol 1984, 173(2):243-251

34. Edman P: A method for the determination of amino acid sequence in peptides. Arch Biochem 1949, 22(3):475.

doi:10.1186/1475-2859-11-97

Cite this article as: Klatt and Konthur: Secretory signal peptide modification for optimized antibody-fragment expression-secretion in Leishmania tarentolae. Microbial Cell Factories 2012 11:97

\section{Submit your next manuscript to BioMed Central and take full advantage of:}

- Convenient online submission

- Thorough peer review

- No space constraints or color figure charges

- Immediate publication on acceptance

- Inclusion in PubMed, CAS, Scopus and Google Scholar

- Research which is freely available for redistribution 